\title{
Physical Properties of Trojan and Centaur Asteroids
}

\author{
M. A. Barucci \\ Observatoire de Paris \\ D. P. Cruikshank \\ NASA Ames Research Center \\ S. Mottola \\ Deutsches Zentrum für Luft- und Raumfahrt, German Aerospace Center \\ M. Lazzarin \\ University of Padova
}

\begin{abstract}
Trojans and Centaurs are primitive, peculiar objects orbiting in the middle solar system. Both groups characteristically have low albedos and red colors. Physical observations of Trojans reveal featureless reddish spectra, implying surfaces probably rich in complex organic solid materials. The interiors are expected to be rich in $\mathrm{H}_{2} \mathrm{O}$ ice and other volatile material. Centaurs have surfaces showing dramatically different spectral reflectances, from neutral to very red. Some spectra are featureless, while others show signatures of water ice, methanol, or other light hydrocarbons. Trojans were formed near Jupiter's orbit, while Centaurs were formed far beyond Jupiter's orbit, but both were formed at low temperatures at which water exists as solid ice.
\end{abstract}

\section{INTRODUCTION}

This chapter concerns the physical properties of two populations of minor bodies: the Trojans and the Centaurs, defined primarily on the basis of their specialized orbital characteristics.

The Trojan asteroids that we consider here are members of the population of objects that are coorbital with Jupiter. Mars is also known to have at least four coorbital asteroids, and the outer planets may have similar companions that have not yet been discovered (Weissman and Levison, 1997; Evans and Tabachnik, 2000), thus expanding the original definition of a Trojan asteroid beyond those that are companions to Jupiter. There are two swarms of jovian Trojans, each consisting of a number of objects that librate about the $L_{4}$ and $L_{5}$ Lagrangian points in Jupiter's orbit, $60^{\circ}$ of heliocentric ecliptic longitude ahead and $60^{\circ}$ behind the planet, and possessing sufficient dynamical stability to survive over the age of the solar system (Marzari and Scholl, 1998).

Because of their special place in the solar system vis-àvis Jupiter, the Trojans are of particular interest and importance in our attempts to understand the origin and evolution of Jupiter and its systems of inner (regular) and outer (irregular) satellites. The surface and internal compositions of the Trojans are expected to reflect the materials and conditions in the solar nebula at the time and location of their accretion. The questions that draw us to a study of the physical properties of these objects, using currently available remote-sensing techniques (spectroscopy, radiometry, and photometry), include whether the conditions of formation were uniform for all Trojans, how genesis of the Trojans was related to the formation of Jupiter and the satellites, and how formation of the Trojans relates to small satellites of the other outer planets.

Over the age of the solar system, the gravitational action of Neptune on the inner regions of the Kuiper Disk has caused an erosion of the Kuiper Disk population, ejecting a large fraction of the bodies that formerly occupied nearcircular orbits in the range of $~ 30-45$-AU heliocentric distance (Duncan et al., 1995; Levison and Weissman, 1999). This process continues at the present time. Most of these bodies have been perturbed to more distant orbits, but some of them are perturbed into orbits that traverse the inner regions of the solar system, giving rise to the short-period comets and to the Centaurs (Levison and Duncan, 1997). The Centaur objects occupy orbits in the region of the outer planets, typically crossing the orbits of those planets. They are dynamically unstable, with dynamical lifetimes of $\sim 10^{6}$ $10^{7} \mathrm{yr}$ before ejection from the planetary region or collision with a planet. The Centaurs are given traditional minorplanet designations, although in terms of their physical properties many of them differ from the main-belt and Trojan asteroids.

Measurements of thermal emission of a few Trojans and the Centaurs indicate that these objects have low surface albedos, in the range of 0.03-0.13 (e.g., Cruikshank, 1977; Fernandez et al., 2002). Various lines of observational evidence suggest that much of the surface material is C-rich, in the form of complex organic polymers, polycyclic aromatic hydrocarbons, and other macromolecular compounds (e.g., Cruikshank and Khare, 2000; Khare et al., 2001). 
Specifically, the occurrence of a vast array of organic solids in the low-albedo nucleus of Comet Halley (e.g., Kissel and Krueger, 1987), as well as those in the black carbonaceous meteorites (Cronin et al., 1988; Cronin and Chang, 1993; Ehrenfreund et al., 2001), support the link between low-albedo surfaces and the presence of $\mathrm{C}$ in many forms. Some combinations of minerals with elemental $\mathrm{C}$ of inorganic origin may be able to match the surface reflectance properties of some of the low-albedo objects in the solar system, as discussed below for the case of Trojan asteroid 624 Hektor.

Thus, while the Trojans probably originated in or near their current heliocentric distance, the Centaurs have clearly come to the planetary region from an external reservoir, where they are presumed to have accreted in the distant solar nebula. Because of the low temperature, even at $5 \mathrm{AU}$ heliocentric distance, both groups are thought to consist of primitive material - that is, material that has been relatively little altered chemically since the accretion epoch. The details of, and degree to which, primitive materials differ in composition between two solar nebula regions at $\sim 5 \mathrm{AU}$ and 30-50 AU are matters of compelling interest, thus motivating us to pursue physical studies of all available objects in both classes.

\section{TROJANS}

As of October 5, 2001, there were 663 known asteroids in the $\mathrm{L}_{4}$ group and 421 in the $\mathrm{L}_{5}$ group. From a wide-field observational survey, Jewitt et al. (2000) estimate that the population of the $\mathrm{L}_{4}$ group is $\sim 1.6 \times 10^{5}$ (objects with radii $\geq 1 \mathrm{~km}$ ), with a combined mass of $\sim 10^{-4} \mathrm{M}_{\oplus}$. The mean collision velocity in the Trojan clouds is about $5 \mathrm{~km} \mathrm{~s}^{-1}$, similar to that in the main belt (Marzari et al., 1996). This occurs because the lower Keplerian velocities at the heliocentric distance of the Trojan clouds are compensated by the higher average inclinations of the Trojans. The intrinsic collision probability for the Trojans is about twice that found in the main belt, which, contrary to what was commonly accepted earlier, leads to a picture of a Trojan population where considerable collisional evolution has taken place. This scenario is compatible with the discovery of dynamical families among the Trojans (Milani, 1994).

\subsection{Rotational Properties}

Shape and angular momentum are acquired during the accretion process, and are affected by the subsequent collisional evolution of the bodies. The characterization of these properties can provide important clues to the history of the Trojans. Lightcurve observations represent the basic tool for determining the rotational properties of asteroids, allowing for the determination of the rotation rate and angular momentum direction, as well as an approximation of body shape.

Early work by Dunlap and Gehrels (1969) on 624 Hektor, the largest member of the Trojan clouds, reveals a body with a very elongated shape. Hartmann et al. (1988) report lightcurve observations for an additional 18 Trojans. Based on these data, the authors suggest that higher than normal lightcurve amplitudes, and therefore considerably elongated shapes, might be characteristic of the Trojans, possibly reflecting a difference in composition and collisional evolution with respect to their counterparts in the main belt. The authors study the distribution of the lightcurve amplitudes of these Trojans by combining them with the available data for the Hilda asteroids in order to enlarge the data sample. The rationale for this choice is based on the consideration that both Trojans and Hildas reside in the so-called outer belt and are comparatively dynamically isolated from the main-belt population. Further, their lower number density and average Keplerian velocity would have protected them from an intense collisional evolution. However, while this argument is certainly true for the Hildas (Dahlgren, 1998), it does not apply to the Trojans, thus illustrating the need for separate analysis of the rotation properties of the two populations.

Zappalà et al. (1989) analyze the rotation rates of a group of 29 objects, consisting of Trojans, Hildas, and Cybeles, and find that the distribution can be fitted with a Maxwellian function. Binzel and Sauter (1992) report new lightcurve observations of Trojans and, based on a total sample of 31 objects, confirm the presence of high lightcurve amplitudes. The authors, however, find that the amplitudes were significantly larger than in the main belt only for objects larger than $\sim 90 \mathrm{~km}$.

This work focuses mainly on obtaining information on amplitudes for the largest possible number of objects, therefore compromising on coverage. As a result, the determinations of rotation periods are not highly reliable, and no conclusions on the rotation period distribution can be drawn. Another problem related to these initial investigations is that both the Trojan and the main-belt comparison samples used were affected by a considerable observational bias tending to favor the determination of high amplitudes and short periods.

The necessity of determining a sizable, reliable, and unbiased sample of rotation periods and amplitudes has motivated a major observational program to systematically explore the rotational properties of Trojans (S. Mottola et al., personal communication, 2001). In this study, new reliable rotation periods and amplitudes have been measured for 72 Trojans down to an absolute magnitude of $\mathrm{H} \sim 10.2$, which, combined with the existing dataset, have resulted in determinations for 75 objects, most of which are in the diameter range $70-150 \mathrm{~km}$. Particular care was taken to avoid the observational bias present in previous surveys. Furthermore, a sample of main-belt asteroids in the same size range was observed, in order to provide a suitable, unbiased comparison sample.

2.1.1. Rotation rates. The distribution of the rotational frequencies, $\mathrm{f}_{\mathrm{T}}$, for 75 Trojans is shown in Fig. 1a. The mean value is $f_{T}=2.14 \pm 0.12 \mathrm{rev} / \mathrm{d}$, which compares to $\mathrm{f}_{\mathrm{M}}=2.26 \pm$ $0.14 \mathrm{rev} / \mathrm{d}$ for the main-belt control sample. The two values fall within $1 \sigma$ of one another, and therefore the means can be considered statistically indistinguishable. 

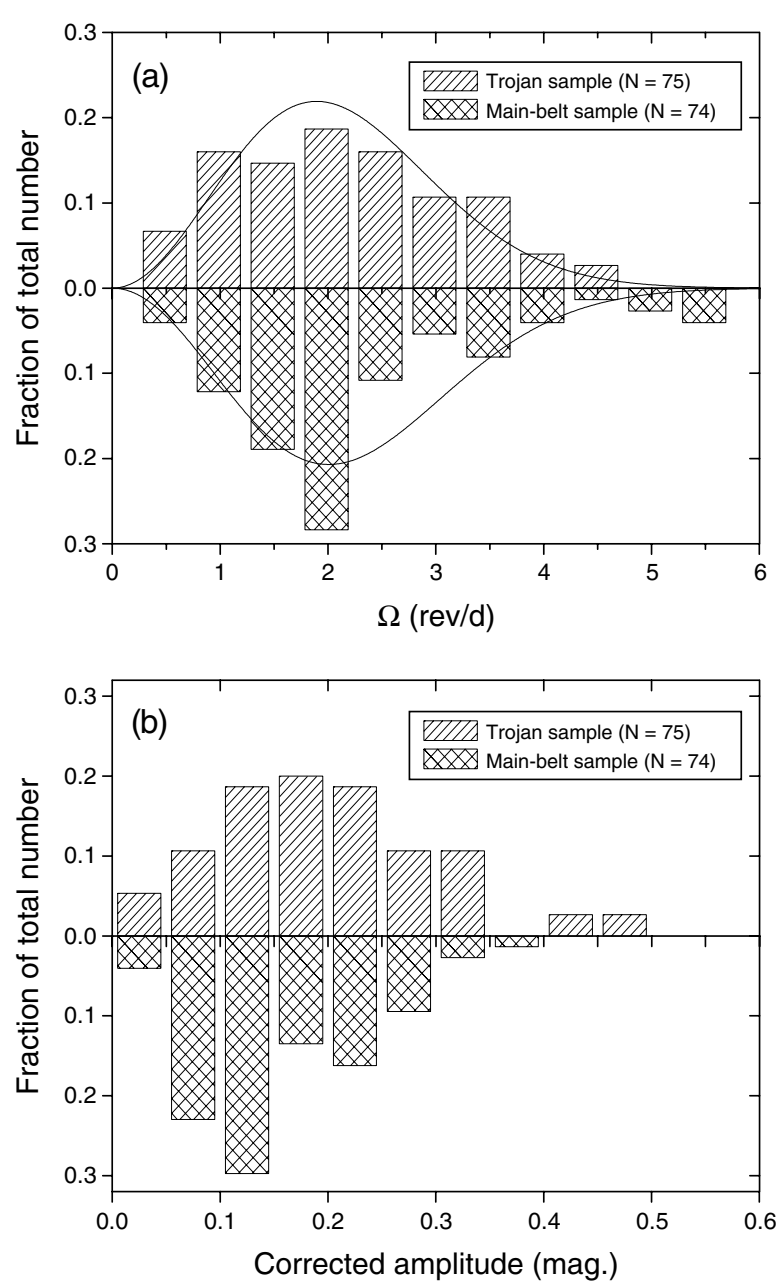

Fig. 1. (a) Distribution of the rotation periods for the Trojan and for the main-belt sample. The best-fit Maxwellian for the two distributions are shown as a solid line. (b) Distribution of the amplitudes reduced to an aspect of $60^{\circ}$ and $0^{\circ}$ phase angle. The average amplitude is $0.198 \pm 0.011$ for the Trojan and $0.155 \pm 0.009$ for the main-belt sample.

The distributions of the spin rates of asteroids have often been compared to single (or linear combinations of) Maxwellian distributions, which are considered to represent, although with some limitations, the rotational state of a collisionally relaxed population (Farinella et al., 1981; Binzel et al., 1989; Fulchignoni et al., 1995). Figure 1a shows the best-fit Maxwellian distribution as a solid curve for the Trojan and main-belt samples respectively. The Trojan distribution is reasonably approximated by a Maxwellian function. On the other hand, the main-belt sample displays an excess of rotators with a period of $\sim 12 \mathrm{~h}$, and a deficiency of rotators in the range of 8-10 h. A Kolmogorov-Smirnov statistical test confirms these findings: The Trojan sample is found compatible with the Maxwellian distribution, while the hypothesis that the main-belt sample is drawn from the best-fit Maxwellian distribution is formally rejected at the 95\% confidence level.
This result suggests that the Trojan population withstood a higher degree of collisional evolution compared to the main belt, which would be consistent with the high values for the intrinsic collisional probability and high average collisional velocity computed for the Trojans.

2.1.2. Lightcurve amplitudes. The amplitude of a lightcurve is an indicator of an asteroid's elongation. The amplitude, however, varies considerably depending on the unknown aspect angle under which the observations are made, with the amplitude being largest with an equatorial aspect and smallest with a polar aspect. When we observe an object at a random epoch, if the spin vectors are distributed isotropically on the celestial sphere, the most probable value for the aspect is $60^{\circ}$. If each object is observed only once, we can directly compare the amplitudes at random epochs. However, if we are confronted with a mixed dataset, where some objects have been observed only once, and some have been observed on several occasions, we have to identify a procedure to select the right amplitudes. Hartmann et al. (1988) select the largest available amplitude for their sample, in an attempt to estimate the maximum lightcurve amplitude. The reason for doing so is that the maximum possible amplitude is directly related to the a:b semiaxis ratio by the relation $\mathrm{A}_{\mathrm{ma}}=2.5 \log$ (a:b) (assuming a triaxial ellipsoid shape).

However, this approach tends to underestimate the maximum amplitudes, and therefore the $a: b$ ratio, for objects that are observed only once. In order to overcome this problem, Binzel and Sauter (1992) propose a scheme for reducing the amplitudes to the aspect of $60^{\circ}$ in case of multiple observations that we have adopted, in slightly modified form, in the following analysis.

Figure 1b shows the distribution of corrected amplitudes of the Trojan and of the main-belt comparison samples. The Trojans appear to have a larger mean amplitude than the main-belt comparison sample. This difference is significant at the $99 \%$ confidence level, with the student's t-test resulting in a probability smaller than $1 \%$ that the two distributions do indeed come from the same population, and the observed difference is only the result of a statistical fluctuation. The shapes of the distributions are also statistically different, with the Kolmogorov-Smirnov test rejecting the null hypothesis (the two datasets coming from the same distribution) at the $95 \%$ confidence level.

Figure 2 shows the lightcurve amplitude vs. the diameter. From this graph no obvious trend of increasing amplitudes with size is recognizable.

2.1.3. Spin axes. The spin axis orientation has been computed for eight Trojan asteroids (S. Mottola et al., personal communication, 2001), two of which belong to the $\mathrm{L}_{4}$ cloud and six to the $\mathrm{L}_{5}$ cloud. The obliquity of the spin appears to be randomly distributed in space, with four of the objects rotating in a prograde and four in a retrograde sense. Even within the limitation of small-numbers statistics, the spin axis distribution appears similar to that of the main belt, which was recently studied by Erikson (2000). Clearly, the possibility that the large observed amplitudes 


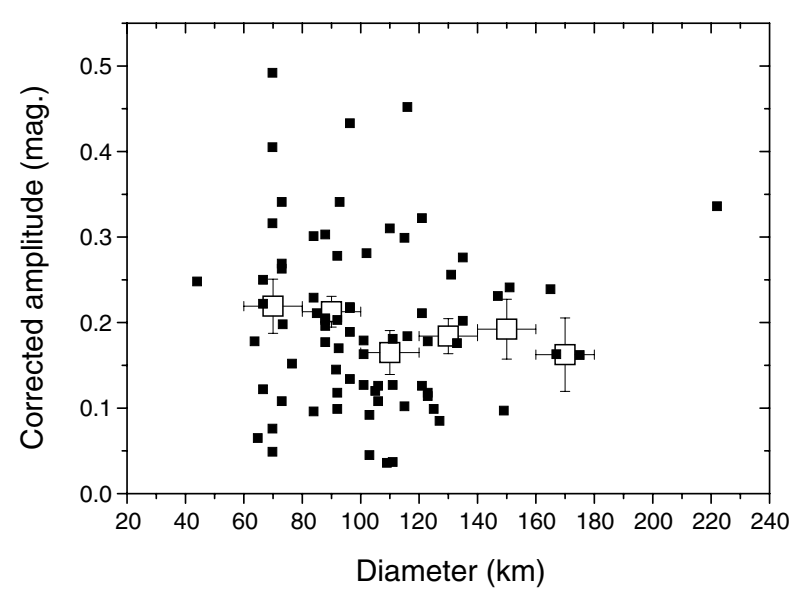

Fig. 2. Amplitude-size distribution of the Trojan sample. Filled squares represent individual asteroids. Empty squares represent the average amplitudes within a $20-\mathrm{km}$ bin. The points at about $45 \mathrm{~km}$ and $220 \mathrm{~km}$ have not been included in the average.

are due to the alignment of the spin axis toward the ecliptic pole, which would favor observations at equatorial aspect, can be ruled out.

\subsection{Taxonomic Classes, Visible and Infrared Spectroscopy}

Only a few of the detected Trojan asteroids have an assigned taxonomical class, but almost all of the classified objects are D-type asteroids; only a few belong to the $\mathrm{P}$ class. D-type objects have very low albedo $(\sim 0.065)$ and are usually characterized by featureless spectra with neutral to slightly red spectra shortward of $0.55 \mu \mathrm{m}$ and very red longward of $0.55 \mu \mathrm{m}$ (Barucci et al., 1987; Tholen and Barucci, 1989; Jewitt and Luu, 1990). In fact, reflectance spectra in the optical region (Jewitt and Luu, 1990; Fitzsimmons et al., 1994; Lazzarin et al.; 1995) and in the NIR (Luu et al., 1994; Dumas et al., 1998; Cruikshank et al., 2001) have not yet shown the clear presence of any feature, and are very similar to the spectra of the nuclei of shortperiod comets and also some Centaurs and KBOs investigated to date.

In particular, the CCD visible spectra of 32 Trojans obtained by Jewitt and Luu (1990) have shown slight differences in spectral behavior, with spectral slopes ranging from nearly neutral $\left(\mathrm{S}^{\prime}=3 \pm 1 \% / 10^{3} \AA\right)$ to very red $\left(\mathrm{S}^{\prime}=25 \pm 1 \%\right.$ / $10^{3} \AA$ ), giving a mean value $\mathrm{S}^{\prime}=9.6 \pm 4.7 \% / 10^{3} \AA$ (Fig. 3 ). The spectra of the 32 Trojans have also been compared with the existing spectra of cometary nuclei in order to study possible relationships between the two groups. The reflectivity gradients of cometary nuclei spectra (mean value $\mathrm{S}^{\prime}=14 \pm$ $5 \% / 10^{3} \AA$ relative to five cometary nuclei) fall in a range similar to that of the spectra of Trojan asteroids. Some of the 32 Trojans were subsequently observed by others (Fitzsimmons et al., 1994; Lazzarin et al., 1995); the slightly different values of $S^{\prime}$ that were found might be associated with different regions of the surfaces of these objects, but also could be attributed to different acquisition and analysis of the data.

In the NIR, the spectra of Trojans usually continue to be reddish (Luu et al., 1994). In particular, spectroscopic observations in the I-J-H-K bands obtained by Dumas et al. (1998) of four Trojans have not revealed any spectral bands, and they all show reddish spectra reminiscent of the spectra of the nuclei of short-period comets. K-band spectra of five Trojans and L-band (3.0-3.5 $\mu \mathrm{m})$ spectra of two Trojans were obtained in 1999 by Emery and Brown (2001). They show the red slope expected for $\mathrm{P}$ and $\mathrm{D}$ asteroids and no clear compositional features. Besides the similarity to shortperiod cometary nuclei, the visible and NIR spectra of the Trojans are similar to the spectra of some KBOs and Centaurs.

Hiroi et al. (2001) have recently report that the reflectance spectrum of the Tagish Lake meteorite is a close match to the spectra of D-type asteroids over the spectral range $0.3-3 \mu \mathrm{m}$. They suggest that this meteorite, an ungrouped C2 carbonaceous chondrite that fell in January 2000, originated from a D-type asteroid. While the overall shape and albedo level of the meteorite spectrum do provide a good match, the spectrum at $3 \mu \mathrm{m}$ shows the presence of hydrous material that is not seen in the spectrum of 624 Hektor, as noted in section 2.3 below.

\subsection{The Case of 624 Hektor}

The D-type asteroid 624 Hektor is the largest of the jovian Trojan population. It is highly elongated, with maximum and minimum dimensions of 300 and $150 \mathrm{~km}$ respectively

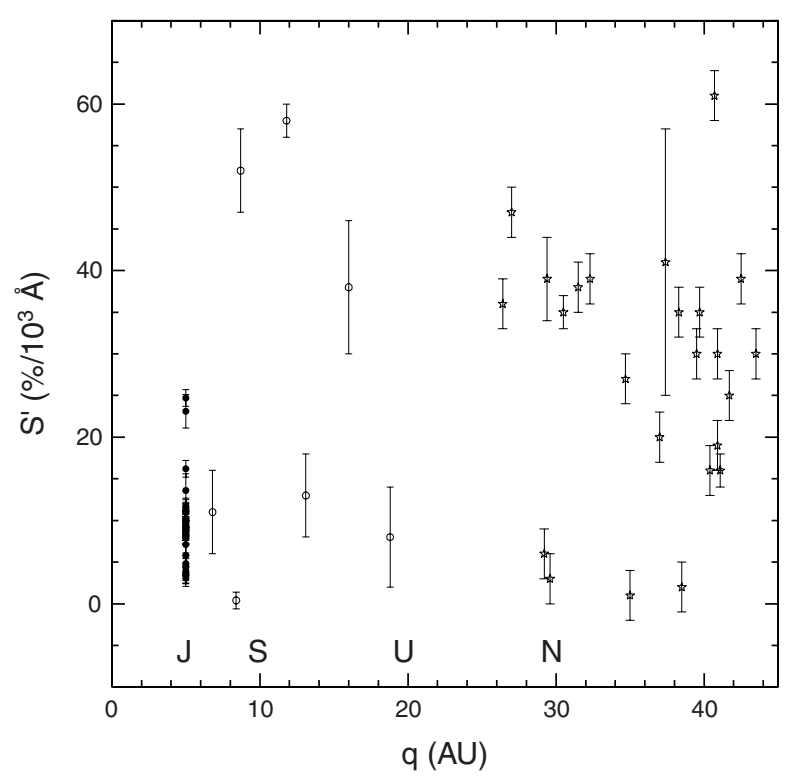

Fig. 3. Visible spectral slope plotted vs. perihelion (q) for Trojans (filled circles), Centaurs (empty circles), and Kuiper objects (stars). The location of the outer planets are marked for reference. 


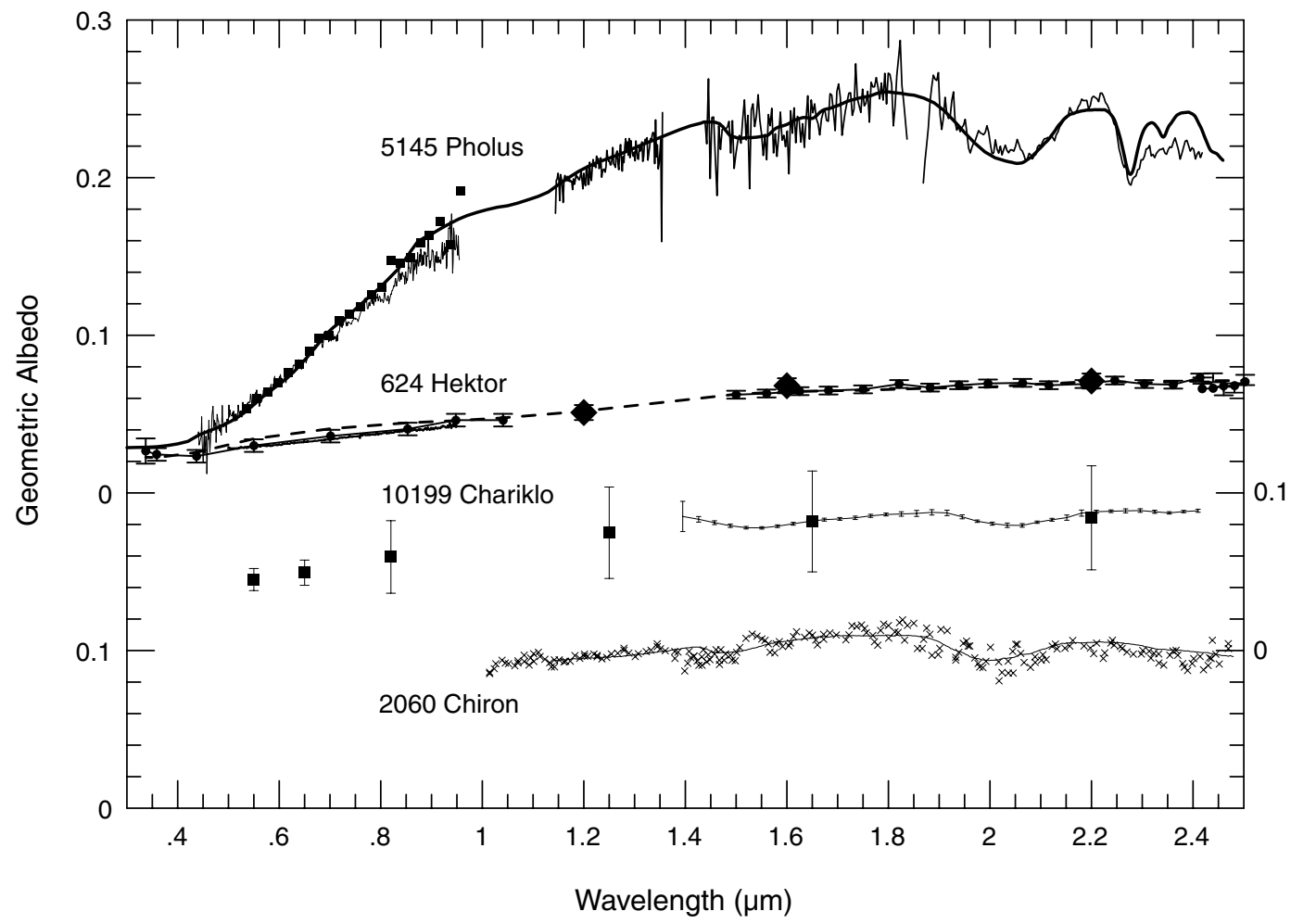

Fig. 4. Reflectance spectra of three Centaurs and Trojan asteroid 624 Hektor. The spectrum of 2060 Chiron is from Luu et al. (2000) and shows a model consisting of $\mathrm{H}_{2} \mathrm{O}$ ice and olivine. The scaling to geometric albedo is approximate. Data for 10199 Chariklo (originally $1997 \mathrm{CU}_{26}$ ) are from Brown et al. (1998); the ordinate for the spectrum of Chariklo is given on the right side of the figure. The 5145 Pholus data and model come from Cruikshank et al. (1998), while the 624 Hektor data and model come from Cruikshank et al. (2001). The ordinate for both Hektor and Pholus is given on the left side of the figure. All three Centaurs show the prominent 2- $\mu$ m $\mathrm{H}_{2} \mathrm{O}$ ice band, with indications of the weaker 1.5- $\mu \mathrm{m}$ band of $\mathrm{H}_{2} \mathrm{O}$ ice. There is no spectral evidence for ice on Hektor.

(Dunlap and Gehrels, 1969; Hartmann and Cruikshank, 1978, 1980). Infrared thermal emission measurements show that Hektor's surface has a low geometric albedo of 0.03 (Cruikshank, 1977; Hartmann and Cruikshank, 1978), while the spectrum [0.48-0.95 $\mu \mathrm{m}$ (Vilas et al., 1993)] and photometry in the eight-color system shows that it is distinctly red (Zellner et al., 1985). The spectrum of Hektor between 0.3 and $1 \mu \mathrm{m}$ (Jewitt and Luu, 1990) and 1.5 and $2.5 \mu \mathrm{m}$ (Cruikshank et al., 2001) shows no distinctive or diagnostic absorption bands that would indicate specific mineral, ice, or organic surface components. Dumas et al. (1998) recorded segments of the spectrum between 0.9 and $2.4 \mu \mathrm{m}$ and found no clear absorption bands. The visible spectrum by Vilas et al. (1993) and Lazzarin et al. (1995) similarly shows no prominent spectral features. While the low albedos and red colors of asteroids and other small bodies in the outer solar system have often been considered suggestive of the presence of macromolecular C-rich surface materials (e.g., Gradie and Veverka, 1980; Cruikshank, 1987, 1997; Cruikshank and Khare, 2000), specific spectral matches have been very hard to establish (e.g., Luи et al., 1994; Moroz et al., 1998).
Cruikshank et al. (2001) extended the spectrum to $3.6 \mu \mathrm{m}$. Using the Hapke scattering theory and the complex refractive indexes of several minerals and organic solids, Cruikshank et al. show that the general features of the Hektor spectrum can approximately be matched with a mixture of Mg-rich pyroxene and elemental C (a mixture of graphite and amorphous $\mathrm{C}$ ). In this model, the pyroxene provides the overall shape of the spectrum (Fig. 4), while the C decreases the albedo to the level observed on Hektor. From the models, the upper limit to the amount of crystalline $\mathrm{H}_{2} \mathrm{O}$ ice (30- $\mu \mathrm{m}$ grains) in the surface layer of Hektor is $\sim 3 \mathrm{wt} \%$; larger amounts mixed in the visible surface layer would show an absorption band at $2 \mu \mathrm{m}$. The upper limit for serpentine, as a representative of hydrous silicates, is much less stringent, at $40 \%$, based on the shape of the spectral region around $3 \mu \mathrm{m}$. Thus, the spectrum at $3 \mu \mathrm{m}$ does not itself preclude the presence of a few weight percent of volatile material in the surface layer of Hektor. All the models require elemental C, which could be of organic or inorganic origin, to achieve the low geometric albedo that matches Hektor.

The general conclusion of this investigation is that the color and low albedo of Hektor may be attributed to a com- 
TABLE 1. Orbital characteristics, diameters, and albedos of Centaurs.

\begin{tabular}{lcccccc}
\hline Name & $\mathrm{q}(\mathrm{UA})$ & $\mathrm{Q}(\mathrm{UA})$ & $\mathrm{E}$ & $\mathrm{i}\left(^{\circ}\right)$ & Diameter $(\mathrm{km})$ & Albedo \\
\hline 2060 Chiron & 8.45 & 18.77 & 0.38 & 6.9 & $180 \pm 10^{*}$ & $0.15 \pm 0.05^{*}$ \\
& & & & & $148 \pm 8^{\ddagger}$ & $0.17 \pm 0.02^{\ddagger}$ \\
5145 Pholus & 8.66 & 31.93 & 0.57 & 24.7 & $190 \pm 22^{\dagger}$ & $0.04 \pm 0.03^{\dagger}$ \\
7066 Nessus & 11.78 & 37.01 & 0.52 & 15.7 & $\approx 75$ & - \\
8405 Asbolus & 6.84 & 29.01 & 0.62 & 17.6 & $66 \pm 4 \ddagger$ & $0.12 \pm 0.03^{\ddagger}$ \\
10199 Chariklo & 13.10 & 18.51 & 0.17 & 23.4 & $302 \pm 30^{*}$ & $0.045 \pm 0.010^{*}$ \\
& & & & & $273 \pm 19 \S$ & $0.055 \pm 0.008^{\S}$ \\
10370 Hylonome & 18.84 & 31.04 & 0.24 & 4.1 & $\approx 150$ & - \\
$1998 \mathrm{SG}_{35}$ & 5.83 & 11.01 & 0.31 & 15.6 & $\approx 35$ & - \\
$2000 \mathrm{QC}_{243}$ & 13.17 & 19.92 & 0.20 & 20.7 & $\approx 190$ & - \\
\hline
\end{tabular}

*Jewitt and Kalas (1998).

$\dagger$ Davies et al. (1993).

$\$$ Fernandez et al. (2002).

$\S$ Altenhoff et al. (2001).

bination of minerals and material of very low albedo and neutral reflectance, and it therefore does not appear to require an organic component. The degree to which this conclusion extends to other D-type asteroids remains to be investigated.

\section{CENTAURS}

Centaurs are minor planets having unstable orbits with semimajor axes between those of Jupiter (5.2 AU) and Neptune (30 AU). Their planet-crossing orbits imply a short dynamical lifetime $\left(10^{6}-10^{7} \mathrm{yr}\right)$ compared to the age of the solar system (Hahn and Bailey, 1990; Asher and Steel, 1993). The origin of the Centaurs is uncertain, but they are thought to have been ejected from the transneptunian belt by planetary perturbations or mutual collisions (Duncan et al., 1995). Levison and Duncan (1997) suggest that these objects could be the source of short-period comets. Later, Levison et al. (2001), on the basis of their numerical model, predict that some Centaurs could have originated in the Oort Cloud. Centaurs seem to be located on a boundary between many solar system populations, and they are important for understanding the dynamical evolution of the outer solar system.

To date, 25 such objects have been discovered, following the continuously updated list from the Minor Planet Center (Marsden, 2001), and the discoveries continue. Although no formal definition exists, Centaurs have been identified as asteroids at the times of discovery, even though 2060 Chiron was subsequently shown to have cometary activity. Jewitt and Kalas (1998) add to the list of Centaurs the comets P/ Oterma and P/Schwassmann-Wachmann 1 because their orbits lie inside the orbits of Jupiter and Neptune. Recently, Marsden (2001) includes Centaurs in a common list with scattered transneptunian objects, arguing that there are no dynamical reasons to make a distinction, but in this paper we will consider the two populations to be well separated.

Jewitt et al. (1996), on the basis of the number of detections in their ecliptic survey, suggest a population of about 2600 Centaurs with diameters greater than $75 \mathrm{~km}$. From the rate of detections by the Spacewatch automated search program, Jedicke and Herron (1997) estimate an upper limit of 2000 Centaurs in the absolute magnitude range $\mathrm{H}<10.5$. This implies that although the density of the population is transient, the Centaurs can be as numerous as the main-belt asteroids over the same size range. Using a wide-field sky survey, Sheppard et al. (2000) estimate that the Centaur population of objects with radius $\geq 1 \mathrm{~km}$ is about $10^{7}$ (if the albedo is 0.04). They predict about 100 Centaurs larger than $50 \mathrm{~km}$ in radius, of which only a few have been found. The current total mass of the Centaurs is estimated to be $\sim 10^{-4} \mathrm{M}_{\oplus}$. From their large survey to search for the brightest transneptunians and Centaurs, Larsen et al. (2001) found a lower density distribution compared to the Jedicke and Herron survey, but within the error bars.

Only a few objects have been observed at thermal and millimetric wavelengths; thus, only a few have well determined diameters and albedos (Table 1). For others, an indication of the diameter can be obtained from the absolute magnitude, assuming a low albedo value $(\sim 0.05)$.

\subsection{The Particular Case of 2060 Chiron}

Chiron was discovered by Kowal (1978). No other asteroids had been discovered at such large distances (orbital semimajor axis $=13.7 \mathrm{AU}$ ), and it was given the name of a Centaur in recognition of its unusual orbit. The orbit was found to be chaotic and subject to strong perturbations by Saturn and Uranus (Oikawa and Everhart, 1979; Scholl, 1979) and thereby typical of short-period comets (Hahn and Bailey, 1990). In 1988, when Chiron was at 13 AU, Tholen et al. (1988) found brightness fluctuations indicating possible cometary behavior of the object; a cometary behavior of Chiron was indicated also by Bus et al. $(1988,1989)$ and Hartmann et al. (1990). A coma was detected for the first time by Meech and Belton (1989).

Chiron's cometary activity produces an unusual longterm brightness variation. Some investigators found that the cometary activity increases as Chiron approaches perihelion, but the brightness diminishes (Lazzaro et al., 1996). Luи 
(1993) analyzed photometric data from 1982 to 1992 showing a series of outbursts, with brightness fluctuations as large as 1 mag occurring on timescales of a year. She concluded that long-term (timescale of $\sim 1 \mathrm{yr}$ ) and short-term (timescale of days) outbursts are the principal causes of the activity of Chiron, even at large distances such as $13 \mathrm{AU}$. Brightness variations were also reported by Bus et al. (1991), and, in a recent analysis, Bus et al. (2001) used archival photographic plates to determine Chiron's brightness between 1969 and 1977. When added to the postdiscovery observations, it is clear that Chiron's brightness does not depend directly on its heliocentric distance; there was an extensive interval of brightening near the most recent aphelion passage.

Lazzaro et al. (1997) investigate changes in Chiron's brightness by performing a photometric survey along the passage of Chiron through perihelion in 1996. The monitoring of Chiron's brightness in 1996 showed that its mean absolute $\mathrm{V}$ magnitude varied by $\sim 10 \%$ in a few months. Parker et al. (1997), using UV data from the Hubble Space Telescope, reveal a $20 \%$ magnitude increase in activity in January and April 1996. Lazzaro et al. (1997) also found short-period activity of the order of hours during one night. A similar burst of short-period activity was also detected in 1989 by Luu and Jewitt (1990) and in 1990 by Buratti and Dunbar (1991).

The synodic rotation period of $5.9180 \pm 0.0001 \mathrm{~h}$ was determined by Bus et al. (1989) and is similar to the value determined by Marcialis and Buratti (1993) and Lazzaro et al. (1997).

Two reflectance spectra in the visible region were obtained in 1996 nearly at the same time as the photometric data by Lazzaro et al. (1997), and they show a quite flat, featureless continuum with a little difference in slope: The reddest corresponds to an increase of the brightness of Chiron. The reflectivity gradient of these two spectra (ranging from $S^{\prime}=2.3 \pm 0.1 \% / 10^{3} \AA$ to $S^{\prime}=0.4 \pm 0.1 \% / 10^{3} \AA$ ) is rather different from the mean-reflectance slope of optical spectra of cometary nuclei, which are usually redder in color $\left(\mathrm{S}^{\prime}=14 \pm 5 \% / 10^{3} \AA\right)$ and is more similar to that of C-type asteroids. A negative reflectivity gradient was also found by Luu (1993), Fitzsimmons et al. (1994), and Barucci et al. (1999). This color peculiarity is not unique to Chiron; Davies et al. (1998) and Barucci et al. (1999) find that the optical reflectivity gradients of the Centaurs are different from one another, indicating a diversity in surface composition probably due to different evolutionary mechanisms. The small variations in the reflectivity gradient $\mathrm{S}^{\prime}$ of Chiron could be due to variations in dust production during episodes of cometary activity. The difference in the reflectivity of Chiron compared to the other Centaurs, which are so far all redder than Chiron, has been attributed (Luu et al., 2000) to cometary activity on Chiron. Such activity would cause a continuous rain of cometary debris on the surface of Chiron, burying a primordial irradiation mantle with unirradiated matter ejected from the interior (Luи et al., 2000).

Chiron is one of the few objects that has shown activity at distances greater than $12 \mathrm{AU}$, and there has always been a debate on the mechanism responsible for the activity and outbursts of these distant bodies. The sublimation of water ice, which drives the activity in nearby comets $(r<3 \mathrm{AU})$, would not occur at Chiron's heliocentric distances. More volatile ices such as $\mathrm{CO}$ or $\mathrm{CO}_{2}$ are probably responsible (Capria et al., 2000). Prialnik et al. (1995) suggest that the outbursts on Chiron could be due to a combination of energy released by the crystallization of amorphous water ice and the release of trapped $\mathrm{CO}$ from a porous matrix of both amorphous and crystalline ice. Gaseous $\mathrm{CO}$ was probably found in Chiron's coma in 1995 by Womack and Stern (1995) via the molecule's $\mathrm{J}=1-0$ rotational transition at $115 \mathrm{GHz}$, but the result still needs to be confirmed. Another molecule that could be in part responsible for the activity on Chiron is CN, found during the outburst of January 29, 1990, by Bus et al. (1991). In particular, Bus et al. (1991) suggest that CO and $\mathrm{CO}_{2}$ would drive the activity, and their escape from the surface of Chiron could be responsible of the release of the parent of the $\mathrm{CN}$.

In spite of the earlier featureless spectra in the visible and NIR, Chiron has recently shown (see Fig. 4) the presence of water ice through the 2- $\mu \mathrm{m}$ absorption band (Foster et al., 1999; Luu et al., 2000). The ice present on the surface of Chiron is very likely mixed with dark impurities, a small amount of which is usually enough to mask the spectral band.

\subsection{Rotational Periods and Lightcurve Amplitudes}

In addition to Chiron, rotational periods have been determined for a few Centaurs:

5145 Pholus has a rotational period of $9.9825 \pm 0.004 \mathrm{~h}$ determined by Buie and Bus (1992) and confirmed with small differences by Hoffmann et al. (1993) and Davies et al. (1998). The lightcurve seems almost symmetric, with an amplitude of $\sim 0.15$ mag. No significant $V-R$ variation is evident with rotation.

8405 Asbolus has been observed photometrically by Brown and Luu (1997), who derive a period of $8.87 \pm 0.02 \mathrm{~h}$ with an amplitude of $\sim 0.34$ mag. Davies et al. (1998), using new data, argue for a slightly longer period (8.9351 \pm $0.0003 \mathrm{~h})$ and larger amplitude $(\sim 0.55 \mathrm{mag})$. Kern et al. (2000), on the basis of spectroscopic observations and on the hypothesis that the lightcurve may be dominated by a relatively bright surface spot, suggest the possibility of a rotational period half of the previous value $(4.47 \mathrm{~h})$.

10199 Chariklo was observed by Davies et al. (1998), who suggest a rotation period close to $24 \mathrm{~h}$ or longer and a very small amplitude. Peixinho et al. (2001) suggest a possible rotational period of $31.2 \mathrm{~h}$ from nine nights of observation, while Alessandrino et al. (personal communication, 2001) propose a very long rotational period of $39 \pm 6 \mathrm{~d}$.

1998 SG $_{35}$ was observed by Green (Davies, 2000) with the preliminary result that the rotational period seems longer than $12 \mathrm{~h}$ and the amplitude larger than 0.04 mag.

$1999 \mathrm{UG}_{5}$ was observed by Gutierrez et al. (2001), who determined a period of $\sim 26.5 \mathrm{~h}$ (but other values are possible) and an amplitude of $0.24 \pm 0.02 \mathrm{mag}$. 
There are no pole inclinations or shape constraints available for these objects. From the amplitude of the lightcurves we can estimate a lower limit on the axis ratio, assuming an ellipsoidal shape with semiaxes $a>b>c$. That limit is $a: b \geq 10^{0.4 \Delta \mathrm{m}}$ if we assume that the lightcurve is influenced only by the shape and not by significant albedo variations. The few observed Centaurs seem to have small amplitudes, except 8405 Asbolus, for which the amplitude is $0.55 \mathrm{mag}$, corresponding to $a: b \geq 1.66$. This elongated shape and its small diameter $(\sim 66 \mathrm{~km})$ could give some indication of the collisional evolution of this object.

\subsection{Colors}

A wide range of broadband colors has been found in the Centaur population. The colors vary from neutral or slightly blue for 2060 Chiron $(\mathrm{V}-\mathrm{R}=0.37)$ to very red for 5145 Pholus (V-R = 0.78). 7066 Nessus has a red color very similar to Pholus in the visible range, but its redness is less evident in the IR region. Laboratory experiences suggest that a red color surface can be produced by the energetic processing (exposure to cosmic rays, solar ultraviolet, corona discharge, and/or ion bombardment) of surface ices, organic solids, and even minerals. Such processing forms a coating of dark materials and a very red spectrum (e.g., Andronico et al., 1987; Thompson et al., 1987).

The color distribution of Centaurs, in the B-V vs. V-R plot (Fig. 5), seems to be bimodal, but the sample is still too small. Two groups are evident: one as Chiron with neutral/ slightly red colors and the other one with much redder colors, more similar to Pholus. As has been demonstrated by Doressoundiram et al. (2001), this dichotomy is further enhanced by the fact that Chiron is an active comet, while no activity has been detected on Pholus, which should have a more pristine surface. In fact, Pholus might possess a thick irradiation mantle that inhibits outgassing of volatiles, while the neutral color on Chiron may be due to outgassing during successive episodes of activity, with a surface most likely dominated by a dust layer created from cometary debris (Luu et al., 2000). Therefore, as Luu et al. (2000) and Doressoundiram et al. (2001) suggest, two groups may exist: one (like Chiron) in which a reddish crust can be removed or processed by activity or impact and the other one (like Pholus) with older surfaces, probably due to their recent escape from the Kuiper Belt.

No correlation has been found between color variations and heliocentric distance and/or diameter (Fig. 3).

\subsection{Spectroscopy}

As has been shown by their color differences, Centaurs have surfaces showing dramatic spectral differences from neutral to very red. In the visible region, Barucci et al. (1999) observe five of them, confirming a great diversity among the reflectances of these objects. They obtain featureless spectra distributed between very flat for 2060 Chiron to very red for Nessus, which appears in this region

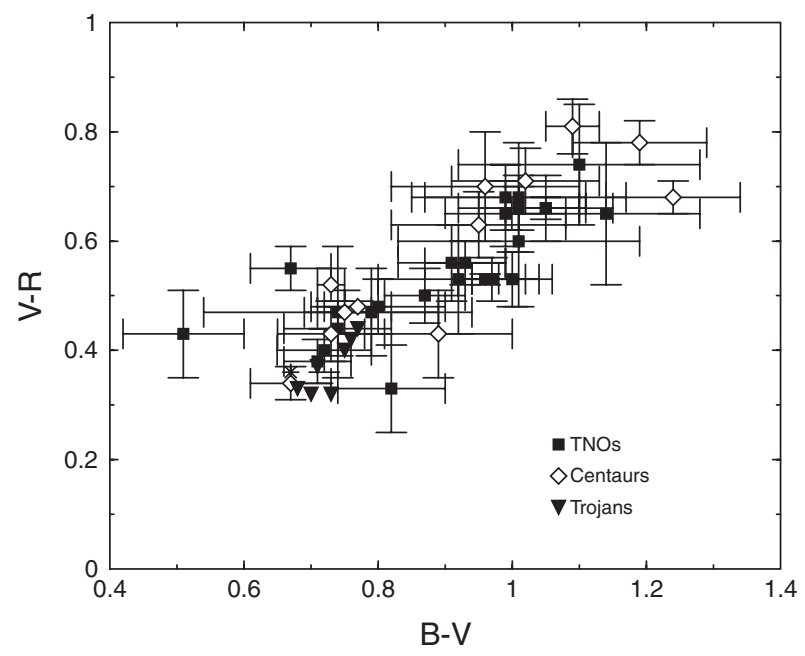

Fig. 5. B-V vs. V-R color of Centaurs (empty diamonds) and Trojans (filled triangle) with comparison of TNOs (filled squares).

to be nearly as red as 5145 Pholus, the reddest object known in the solar system so far.

In the IR region, some spectra are featureless, while some others show signature of water ice, methanol, or other light hydrocarbon ices. Very few of these objects have been well studied or rigorously modeled in the visible and NIR (Fig. 4).

5145 Pholus was observed in the visible and NIR by several authors and a synthesis was presented by Cruikshank et al. (1998). The NIR spectrum shows a strong absorption band at 2.04 and at $2.27 \mu \mathrm{m}$. The large band at $2.04 \mu \mathrm{m}$ is typical of water ice while the other at $2.27 \mu \mathrm{m}$ might be due to methanol ice. Cruikshank et al (1998) modeled the complete spectrum of Pholus from 0.4 to $2.5 \mu \mathrm{m}$ with Hapke scattering theory, interpreting the extraordinary red color and the rich NIR features as due to the surface presence of common silicate olivine, a complex organic solid (tholin), water ice, methanol $\left(\mathrm{CH}_{3} \mathrm{OH}\right)$ (or another light hydrocarbon), and $\mathrm{C}$. They conclude that Pholus is a primitive object hat has not yet been substantially processed by solar heating and probably is a nucleus of a large comet that has never been active.

8405 Asbolus was observed by Brown (2000) and Barucci et al. (2000). Both spectra show a lack of spectral signatures, in particular no absorptions due to water ice (bands at 1.5 and $2 \mu \mathrm{m})$. As in the case of Pholus, Barucci et al. (2000) model the complete spectrum of Asbolus from 0.4 to $2.3 \mu \mathrm{m}$. Due to the difficulty in interpreting the spectral behavior of these objects, two models are proposed. One model, with a spatial mixture of $50 \%$ amorphous $\mathrm{C}$ plus $50 \%$ kerogen, can reproduce well the shape of the spectrum, but not below $0.5 \mu \mathrm{m}$. To investigate the limit of the abundance of water ice, another model, with $91.5 \%$ amorphous C, $7.5 \%$ tholin, and $1 \%$ water ice, was proposed. Depending on the grain size, no more than a few percent of the sur- 
face can be covered with pure water ice in order to keep the strong $2-\mu \mathrm{m}$ band below the level of the noise. Kern et al. (2000) obtained a 1-2- $\mu$ m spectra using the Hubble Space Telescope, revealing a significantly inhomogeneous surface characterized by water ice mixed with unknown low-albedo constituents. The spectrum was initially featureless, but during an interval of $1.7 \mathrm{~h}$, the spectrum developed a strong absorption band at the approximate location of the $1.5-\mu \mathrm{m}$ $\mathrm{H}_{2} \mathrm{O}$ band. Kern et al. (2000) explained this with the possibility that an impact has penetrated the object's crust, exposing the underlying ice in the surface region that rotated into view during the observations. Romon-Martin et al. (2001) reobserved Asbolus at VLT (ESO, Chile) obtaining five IR spectra covering the full rotational period. The new data show flat spectra with no variation in the rotation and no presence of ice absorption.

10199 Chariklo was observed by Brown and Koresko (1998) and Brown et al. (1998). The IR spectra show clear evidence of water ice. The spectrum was modeled by Brown and Koresko (1998) by distinct surface areas of a dark, neutral surface and 3\% water ice. Brown et al. (1998) made a model fit assuming an intimate mixture of red-colored material and higher percentage of water ice, probably in an amorphous state.

10370 Hylonome has been observed by Romon-Martin et al. (personal communication, 2001) and on the basis of preliminary results, the spectrum seems featureless.

Unfortunately, these objects are faint and even observations with the largest telescopes (Keck and VLT) do not generally yield good quality spectra. Interpretation is also very difficult because the behavior of the spectra depends on the choice of many parameters. The uncertainty in determining quantitative abundance on the surfaces is large and the derived models are not unique. Furthermore, the number of plausible materials (ices, organic solids, minerals) that can be incorporated into the scattering models is small because so few such materials have had their complex refractive indexes measured in the laboratory.

\section{RELATIONS TO OTHER SMALL BODIES}

\subsection{Relationship of Centaurs and Trojans to Icy Satellites of the Planets}

The giant planets have many satellites, the largest of which all show spectroscopic evidence for $\mathrm{H}_{2} \mathrm{O}$ and/or other ices on their solid surfaces (the case of Titan is ambiguous). All the regular satellites (low-inclination, near-circular, prograde orbits) of radius $>240 \mathrm{~km}$ have surface ices, and a number of other smaller satellites also show ice bands in the NIR spectra. Among the irregular satellites (highinclination, elliptical, retrograde or prograde orbits), Nereid (Neptune) and Phoebe (Saturn) have detectable ice bands (Brown et al., 1999b; Brown, 2000; Owen et al., 1999). The irregular satellites, in particular, tend to be low-albedo objects, and their ice bands (when detected) are weak, indicating that the ice is mixed at the granular level with low- albedo minerals, C, organic solids, or some combination of the three. Irregular satellites are also thought to be captured objects that originated elsewhere in the solar nebula. It is possible, perhaps likely, that they were captured after extraction from the Kuiper Disk.

The relationship of the Trojan asteroids to icy satellites is not known. The Trojans have very low albedos, consistent with the C-, P-, and D-type asteroids in the main belt and with many of the small outer satellites of Jupiter and the other giant planets. They represent objects that accreted in the zone around or beyond the orbit of Jupiter, and the heliocentric distance they currently occupy marks the inner boundary of the zone of long-term stability for surface exposures of water ice. There are no reported detections of water or other ices in any Trojan spectra (Jewitt and Luu, 1990; Barucci et al., 1994; Dumas et al., 1998), although there may be ices below the surface that are accessible to remote-sensing observation. Neither do we have any measurements of the densities of Trojan asteroids from which the interior composition might be inferred. The work of Cruikshank et al. (2001) on 624 Hektor noted above indicates that hydrous minerals might be present in the surface material and remain undetected with the quality of NIR spectroscopic data we now have. Indeed, the spectral feature at 0.7 and $3 \mu \mathrm{m}$ identified in the spectra of many low-albedo asteroids of the C, G, and F classes in the main belt (Jones et al., 1990; Vilas et al., 1993; Barucci et al., 1998) are associated with hydrous minerals. Hydrous minerals, if present, might indicate the presence of liquid ice that originated through the heating of interior ice in an earlier epoch, or they might indicate the incorporation of materials that were serpentinized by other processes (Reitmeijer and Nuth, 2000, 2001).

Hartmann (1980) noted that planetesimals that formed in Jupiter's region of the solar nebula were probably composed of a mixture of roughly equal amounts of water ice and stony material; the amount of organic solids in this mix could be significant. Material condensing to form planetesimals in the region of Jupiter was subjected to temperatures above $40 \mathrm{~K}$ (Lunine et al., 2000); if the temperature were as high as $55 \mathrm{~K}$, the volatile molecules $\mathrm{N}_{2}, \mathrm{CO}$, and $\mathrm{CH}_{4}$ were not efficiently trapped and incorporated into the planetesimals, but refractory organics, $\mathrm{NH}_{3}$, and compounds of other heavy elements were trapped (Bar-Nun and Kleinfeld, 1989). The surface layers of solid bodies at Jupiter's heliocentric distance are much warmer than $55 \mathrm{~K}$ in the present epoch; average surface temperatures range from $\sim 89$ to $100 \mathrm{~K}$ for objects with geometric albedos 0.4 and 0.04 respectively.

The visible surface of an initially ice-rich body in the Trojan population, or a small ice-rich satellite of Jupiter, could be transformed into a low-albedo surface by the impact gardening and selective removal of the volatile component, leaving a buildup of the rocky and organic solid material (Hartmann, 1980). The eruption of volatile-rich (primarily water) magmas onto the impacted surface following thermal disturbances would serve to redeposit wa- 
ter on the surfaces, but the long-term effect might produce a net darkening and devolatilization of the surface layer that is observed with optical and IR remote-sensing techniques. As noted above, Luu et al. (2000) predict the opposite effect (surface brightening) from cometary activity on Chiron.

Other complex mechanisms might also darken the uppermost surface of an icy-rich body. In particular, the darkening of ices containing simple organic molecules by cold plasma irradiation (Thompson et al., 1987) and ion bombardment (Andronico et al., 1987) has been demonstrated in the laboratory.

\subsection{Relations to Kuiper Belt Objects}

The transneptunian objects, also called Kuiper Belt objects, represent the new frontier of our solar system. More than 500 objects have been discovered up to the present; these represent a small sample of a much larger population of icy planetesimals orbiting at the outer edge of the solar system. They are expected to be the best-preserved fossils of the protoplanetary disk, as they have been formed at very low temperatures $(\sim 40 \mathrm{~K})$ and are believed to be the remnants of solar system formation. Collisions and irradiation have reworked their surfaces, especially in the inner part of the belt, and extensive cratering can be expected to characterize their surfaces (Durda and Stern, 2000).

The physical and chemical properties of the Kuiper objects are still poorly known due to their faintness. Spectroscopic data in the visible and the NIR are available for only a few of these objects. The spectra range from neutral to very red with behavior very similar to that of Trojans and Centaurs (Fig. 3). Most of the information on the surface composition comes from studies in the NIR. Few Kuiper objects have been observed in the NIR, and although the spectra show a very low $\mathrm{S}: \mathrm{N}$, their surface characteristics show a wide diversity: $1996 \mathrm{TL}_{66}$ (Luu and Jewitt, 1998) as well as $2000 \mathrm{~EB}_{173}$ (Brown et al., 2000; and Licandro et al., 2001; Jewitt and Luu, 2001) have flat featureless spectra similar to that of dirty water ice, while $1996 \mathrm{TO}_{66}$ shows an inhomogeneous surface with the presence of small amounts of water ice mixed with other minor components (Brown et al., 2000). 1993 SC, observed by Brown et al. (1997), shows features that may be due to hydrocarbon ices, with a general red behavior suggesting the presence of more complex hydrocarbons, while the spectrum of 1996 SC did not show any similar features when observed by Jewitt and Luu (2001) with the same telescope. In contrast, $1999 \mathrm{DE}_{9}$ shows solid-state absorption features near 1.4, 1.6, 2.00, and probably at $2.25 \mu \mathrm{m}$ (Jewitt and Luu, 2001). This spectral diversity is confirmed by photometric observations of a larger sample. TNO colors exhibit wide diversity, with quasicontinuum color variation ranging from neutral to very red. Barucci et al. (2001) apply for the first time a statistical analysis of B-V, V-R, V-I, and V-J colors of TNO and Centaur populations. The general results show that the sample of objects can be divided into a few groups with spectra ranging from neutral to very red. The quasicontinuum color variation of TNOs can be a consequence of collisions at all scales that resurface the body by fresh debris and of space weathering.

Centaurs appear to have very similar spectral and color characteristics to those of the TNOs (Fig. 3), supporting the hypothesis that Centaurs are ejected from the Kuiper Belt by planetary scattering; this is the strongest observational argument for a common origin. Centaur colors are more similar to the neutral and to the reddest TNOs, while the Trojans are more similar to the neutral/red group (Fig. 5).

The rotational properties of those few Centaurs and Kuiper Belt objects for which the determinations have been made seem to be similar to those of the Trojans and mainbelt asteroids.

\section{CENTAURS AND THEIR EVOLUTION FROM THE INTERSTELLAR MEDIUM}

Notwithstanding the mechanisms for surface modification noted above, the surface composition of a small (undifferentiated) outer solar system body should to some degree be indicative of bulk composition.

In order to understand the compositions and structures of the Centaurs, we consider the problem from the viewpoint of the condensation of solid material, originally from the interstellar medium, in the regions of the solar nebula beyond Neptune. This is the essence of the problem of the origin and compositions of the short-period comets that arise from the Kuiper Belt. In principle, knowledge gained about the interior and surface compositions of comets can be applied more or less directly to the Centaur objects, bearing in mind the disparity in size between typical comets (1$10 \mathrm{~km})$ and Centaur objects discovered up to the present ( $40 \mathrm{~km}$ and greater).

Objects in the Kuiper Disk are thought to have condensed from the solar nebula in the zone from 40-60 AU where they presently reside. The temperature at the time of condensation of these objects may well have been lower than $40 \mathrm{~K}$, thus trapping volatile molecules and heavy noble gases, as well as organic materials that were largely unaltered from their original state in the interstellar medium (Lunine et al., 2000). The degree of processing of interstellar solid material in the outer solar nebula is uncertain. As Lunine et al. (2000) have noted, planetesimals formed in a disk that was embedded within the nascent molecular cloud, and it is reasonable to expect a continuum of processing of material between the cloud and the protoplanetary disk. Certainly the water abundance in the nebula was both spatially and temporally variable during the condensation of the planetesimals.

The nuclei of comets, and, by inference, the Kuiper Belt objects and Centaurs, are assemblages of ices, organic solids, and silicate minerals that solidified in a variety of different environments; they are therefore presumed to be in a state of chemical disequilibrium. When they accreted in the outer solar nebula, they incorporated both unaltered material from the nascent molecular cloud and material that had undergone some degree of chemical and thermal pro- 


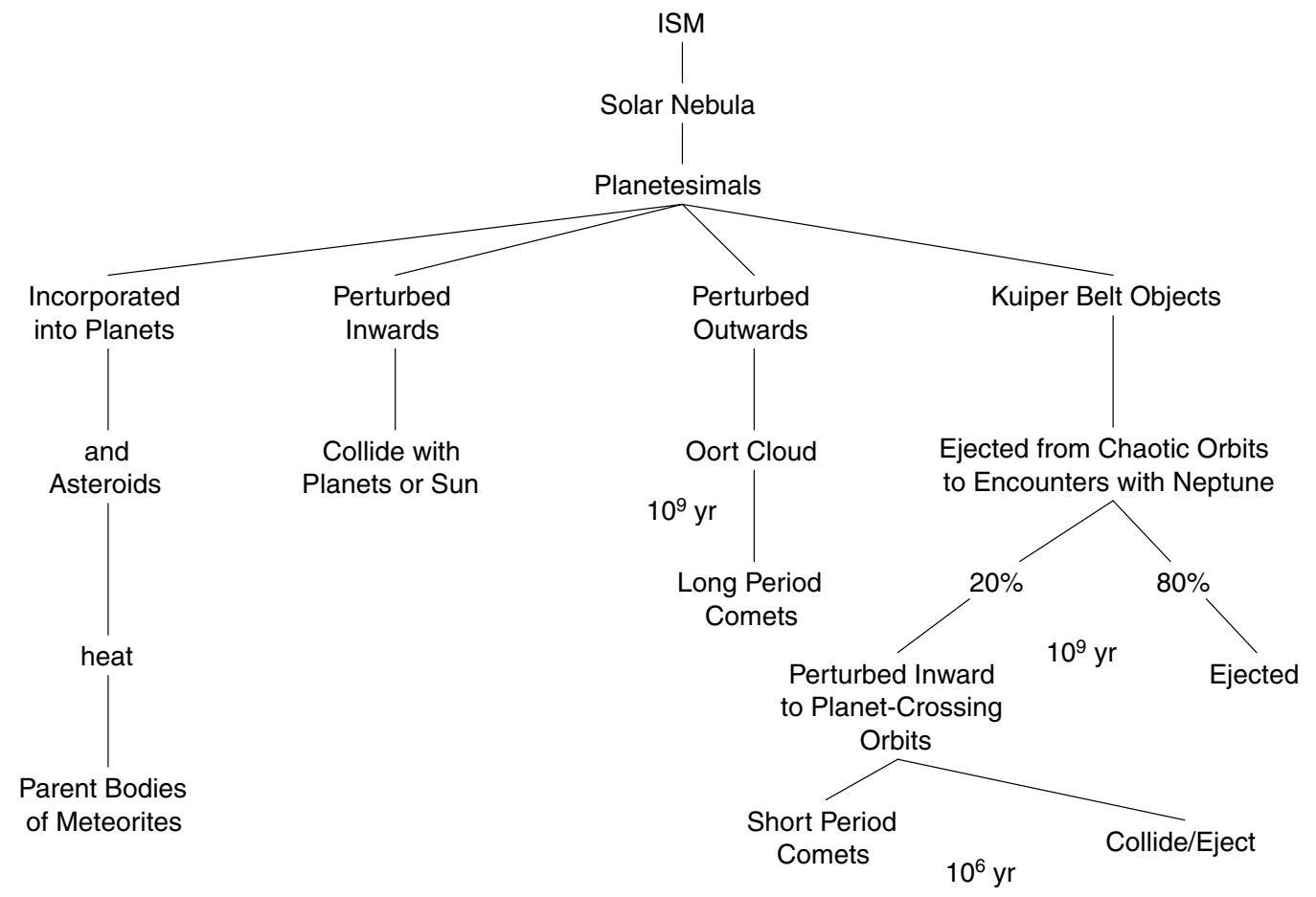

Fig. 6. A scenario for the evolution of the planetesimals in the solar nebula. The approximate dynamical lifetimes in years for some stages in the development are shown. Adapted from Cruikshank (1997).

cessing in the solar nebula. On the basis of the ortho/para ratios of $\mathrm{H}$ in water, measured in several comets, the accretion temperature of cometary ices is estimated to lie in the range 25-35 K (Irvine et al., 2000). The disequilibrium conditions of formation and accretion of cometary materials appear to be reflected in the variety of (surface) compositions on the small number of Kuiper Belt objects and Centaurs for which such information presently exists (e.g., Brown et al., 1997, 1998, 1999a, 2000; Brown, 2000).

How can we integrate these basic ideas into a scenario that embraces the evolution of material from the molecular cloud to the solid bodies we find in the modern solar system? In Fig. 6 we trace the development of material from the interstellar medium into the modern solar system in a very schematic way by following four pathways. One of these pathways, representing the formation and accretion of planetesimals in the inner solar system, leads to asteroids with their wide range of compositions. Another important pathway follows planetesimals in the outer solar nebula to the formation of the Kuiper Disk, from which encounters with Neptune disperse some of these bodies into the planetary region. This extraction from the Kuiper Disk occurs on approximately $10^{9}$-yr timescales, but those objects in planetcrossing orbits (the Centaurs and short-period comets) have dynamical lifetimes of only $\sim 10^{6} \mathrm{yr}$ before they are ejected, disintegrate, or collide with the Sun or a planet.

By some other unclear pathway, not shown in the figure, icy or rocky bodies are trapped in orbits around planets to become the irregular satellites that each of the giant planets seems to have. The pathway to the formation of the jovian Trojan asteroids is also unclear, leaving us to wonder if the Trojans are more compositionally similar to the objects in the outer main belt or to the irregular satellites of Jupiter and Saturn. Unfortunately, the information contained in their surface spectral reflectances appears to be very meager and somewhat ambiguous. In any event, the jovian Trojans occupy a transition zone between these populations, and further clarification of their physical properties will eventually lead to a much better understanding of their origin.

\section{CONCLUSIONS}

Trojans and Centaurs are very interesting populations within the solar system. Trojan asteroids seem to possess the necessary dynamic stability for survival over the age of the solar system (Levinson et al., 1997), and even if the origin of Trojans is still unclear, they surely formed in a region of the solar nebula rich in frozen volatiles. Moreover, it is generally believed that they did not suffer selective induction heating that produced aqueous alteration processes, and probably they still contain frozen water in their interior. The population has undergone a collisional evolution as least as intense as the one that took place in the main belt. This picture is in line with the random orientation of the spin axes of the Trojans and with the distribution of their rotation rates being close to a Maxwellian. The Trojans have on average larger lightcurves amplitudes compared to similarly sized main-belt objects, suggesting that the Trojans 
have more elongated shapes. Although this feature is not yet understood, possible explanations include a different response to collisions, probably due to their different composition and/or shape exaggeration induced by volatile sublimation in extinct cometary nuclei.

If the Trojans formed near or beyond Jupiter's orbit, their temperatures could be low enough for water to have existed always as solid ice. The hypothesis that Trojans could possess ice-rich interiors similar to the cometary nuclei is not contradicted by any available observations. Unfortunately, physical observations provide only limited clues about the source of Trojans.

The Centaurs are located in unstable orbits with short dynamical lifetimes. They have also suffered many collisions but less intensely than Trojans and probably at lower velocities. The presence of the irradiation mantle on the surface of the Centaurs (Luu and Jewitt, 1996) would explain the redness of the spectra of these objects, and their diversity in colors could be probably explained by removal, with different degrees, of the irradiation material exposing primordial ices. However, the slightly neutral spectrum of Chiron distinguishes itself from the behavior of the other Centaurs. Luи et al. (2000) believe that the variable spectrum of the object, the revealed presence of water ice and persistent cometary activity would indicate that the surface is not dominated by an irradiation mantle but by a layer of cometary debris, not uniformly distributed on the surface, that was formed by the sublimation of the ices like $\mathrm{CO}, \mathrm{N}_{2}$, etc. On the contrary, an object like Pholus showing a very red color, absorption features probably due to hydrocarbons materials, and the absence of cometary activity is compatible with a surface covered by an irradiation mantle ( $\mathrm{Luu}$ et al., 2000). They also predict that water ice has to exist on Centaur surfaces and should be ubiquitous among objects originating in the Kuiper Belt; its detectability and possible cometary activity is determined by the water-ice surface coverage.

Outward migration of the planets after their formation and large-scale impacts may have modified the surface composition of objects in the outer solar system. The spectral behavior of Trojans is similar to those of some KBOs, Centaurs, and short-period cometary nuclei. It could be argued that Trojans also have irradiation mantles. However, as opposed to some Centaurs and KBOs, which have shown some spectral features, the lack of any detectable spectral features in the spectra of Trojans could be due to a high level of alteration (dehydrogenation) by solar radiation of the organics present on the surface.

In conclusion, low-albedo objects, in particular Trojans and Centaurs that formed at larger heliocentric distance, have been less thermally processed in comparison with main-belt asteroids and therefore may have better preserved primordial materials. These small bodies can still contain a considerable amount of information about some primordial processes that governed the evolution of the early solar system. The available observations do not allow us at the moment to constraint the evolution of these populations, and for this reason many more NIR and infrared observations are needed to study the physical properties and the composition of these objects. New generation telescopes, and in particular a SIRFT (Space Infrared Telescope Facility) will allow new discoveries and revelations fundamental for the understanding of these small bodies.

\section{REFERENCES}

Altenhoff W. J., Menten K. M., and Bertoldi F. (2001) Size determination of the Centaur Chariklo from millimeter-wavelength bolometer observations. Astron. Astrophys., 366, L9-L12.

Andronico G., Baratta G. A., Spinella F., and Strazzulla G. (1987) Optical evolution of laboratory-produced organics: Applications to Phoebe, Iapetus, outer belt asteroids, and cometary nuclei. Astron. Astrophys., 184, 333-336.

Asher D. J. and Steel D. I. (1993) Orbital evolution of the large outer solar system object 5145 Pholus. Mon. Not. R. Astron. Soc., 263, 179-190.

Bar-Nun A. and Kleinfeld I. (1989) On the temperature and gas composition in the region of comet formation. Icarus, 80, 243253.

Barucci M. A., Capria M. T., Coradini A., and Fulchignoni M. (1987) Classification of asteroids using G-mode analysis. Icarus, 72, 304-324.

Barucci M. A., Lazzarin M., Owen T., Barbieri C., and Fulchignoni M. (1994) Near-infrared spectroscopy of dark asteroids. Icarus, 110, 287-291.

Barucci M. A., Doressoundiram A., Fulchignoni M., Florczak M., Lazzarin M., Angeli C., and Lazzaro D. (1998) Search for aqueously altered materials on asteroids. Icarus, 132, 388-396.

Barucci M. A., Lazzarin M., and Tozzi G.P. (1999) Compositional surface variety among the Centaurs. Astron. J., 117, 1929-1932.

Barucci M. A., de Bergh C., Cuby J.-G., Le Bras A., Schmitt B., and Romon J. (2000) Infrared spectroscopy of the Centaur 8405 Asbolus: First observations at ESO-VLT. Astron. Astrophys., 357, L53-L56.

Barucci M. A., Fulchignoni M., Birlan M., Doressoundiram A., Romon J., and Boehnhardt H. (2001) Analysis of Trans-Neptunian and Centaur colours: Continuous trend or grouping? Astron. Astrophys., 371, 1150-1154.

Binzel R. P. and Sauter L. M. (1992) Trojan, Hilda, and Cybele asteroids: New lightcurve observations and analysis. Icarus, 95, 222-238.

Binzel R. P., Farinella P., Zappalà V., and Cellino A. (1989) Asteroid rotation rates: Distributions and statistics. In Asteroids II (R. P. Binzel et al., eds), pp. 298-315. Univ. of Arizona, Tucson.

Brown M. E. (2000) Near-infrared spectroscopy of Centaurs and irregular satellites. Astron. J., 119, 977-983.

Brown M. E. and Koresko C. D. (1998) Detection of water ice on the Centaur 1997 CU26. Astrophys. J. Lett., 505, L65-L67.

Brown M. E., Blake G. A., and Kessler J. E. (2000) Near-infrared spectroscopy of the bright Kuiper Belt object 2000 EB173. Astrophys. J. Lett., 543, L163-L165.

Brown R. H., Cruikshank D. P., Pendleton Y. J., and Veeder G. (1997) Surface composition of Kuiper Belt object 1993 SC. Science, 276, 937-939.

Brown R. H., Cruikshank D. P., Pendleton Y., and Veeder G. J. (1998) Identification of water ice on the Centaur $1997 \mathrm{CU}_{26}$. Science, 280, 1430-1432.

Brown R. H., Cruikshank D. P., and Pendleton Y. J. (1999a) Water ice on Kuiper Belt object 1996 TO66. Astrophys. J. Lett., 519, L101-L104. 
Brown R. H., Cruikshank D. P., Pendleton Y. J., and Veeder G. J. (1999b) Water ice on Nereid. Icarus, 139, 374-378.

Brown W. R. and Luu J. X. (1997) CCD photometry of the Centaur 1995 GO. Icarus, 126, 218-224.

Buie M. W. and Bus S. J. (1992) Physical observations of 5145 Pholus. Icarus, 100, 288-294.

Buratti B. J. and Dunbar R. S. (1991) Observation of a rapid decrease in the brightness of the coma of 2060 Chiron in 1990 January. Astrophys. J. Lett., 266, L47-L49.

Bus S. J., Bowell E., and French L. M. (1988) (2060) Chiron. IAU Circular 4684.

Bus S. J., Bowell E., Harris A. W., and Hewitt A. V. (1989) 2060 Chiron: CCD and electronographic photometry. Icarus, 77, 223-238.

Bus S. J., A'Hearn M. F., Schleicher D. G., and Bowell E. (1991) Detection of CN emission from 2060 Chiron. Science, 251, 774-777.

Bus S. J., A'Hearn M. F., Bowell E., and Stern S. A. (2001) (2060) Chiron: Evidence for activity near aphelion. Icarus, 150, 94103.

Capria M. T., Coradini A., De Sanctis M. C., and Orosei R. (2000) Chiron activity and thermal evolution. Astron. J., 119, 31123118.

Cronin J. R. and Chang S. (1993) Organic matter in meteorites: Molecular and isotopic analyses of the Murchison meteorite. In The Chemistry of Life's Origins (J. M. Greenberg et al., eds.), pp. 209-258. Kluwer, Dordrecht.

Cronin J. R., Pizzarello S., and Cruikshank D. P. (1988) Organic matter in carbonaceous chondrites, planetary satellites, asteroids and comets. In Meteorites and the Early Solar System (J. F. Kerridge and M. S. Matthews, eds.), pp. 819-857. Univ. of Arizona, Tucson.

Cruikshank D. P. (1977) Radii and albedos of four Trojan asteroids and Jovian satellites 6 and 7. Icarus, 30, 224-230.

Cruikshank D. P. (1987) Dark matter in the solar system. $A d v$. Space Res., 7, 109-120.

Cruikshank D. P. (1997) Organic matter in the solar system: From the meteorites to the Kuiper Belt. In From Stardust to Planetesimals (Y. J. Pendleton and A. G. G. M. Tielens, eds.), pp. 315-333. ASP Conference Series 122.

Cruikshank D. P. and Khare B. N. (2000) Planetary surfaces of low albedo: Organic material throughout the solar system. In Bioastronomy '99 - A New Era in Bioastronomy (G. A. Lemarchand and K. J. Meech, eds.), pp. 253-261. ASP Conference Series 213.

Cruikshank D. P., Roush T. L., Bartholomew M. J., Geballe T. R., Pendleton Y. J., White S. M., Bell J. F. III, Davies J. K., Owen T. C., de Bergh C., Tholen D. J., Bernstein M. P., Brown R. H., Tryka K. A, and Dalle Ore C. M. (1998) The composition of Centaur 5145 Pholus. Icarus, 135, 389-407.

Cruikshank D. P., Dalle Ore C. M., Roush T. L., Geballe T. R., Owen T. C., de Bergh C., Cash M. D., and Hartmann W. K. (2001) Constraints on the composition of Trojan asteroid 624 Hektor. Icarus, 153, 348-360.

Dahlgren M. (1998) A study of Hilda asteroids III. Collision velocities and collision frequencies of Hilda asteroids. Astron. Astrophys., 336, 1056-1064.

Davies J. K. (2000) Physical characteristics of Trans-Neptunian objects and Centaurs. In Minor Bodies in the Outer Solar System (A. Fitzsimmons et al., eds.), pp. 9-24. Springer-Verlag, Berlin.

Davies J., Spencer J., Sykes M., Tholen D., and Green S. (1993) 5145 Pholus. IAU Circular 5698.
Davies J. K., McBride N., Ellison S. E., Green S. F., and Ballantyne D. (1998) Visible and infrared observations of six Centaurs. Icarus, 134, 213-227.

Doressoundiram A., Barucci M. A., Romon J., and Veillet C. (2001) Multicolour photometry of Trans-Neptunian objects. Icarus, 154, 277-286.

Dumas C., Owen T., and Barucci M. A. (1998). Near-infrared spectroscopy of low-albedo surfaces of the solar system: Search for the spectral signature of dark material. Icarus, 133, 221-232.

Duncan M., Levison H. F., and Budd S. M. (1995) The dynamical structure of the Kuiper Belt. Astron. J., 110, 3073-3081.

Dunlap J. L. and Gehrels T. (1969) Minor planets. III. Lightcurves of a Trojan asteroid. Astron. J., 74, 797-803.

Durda D. D. and Stern S. A. (2000) Collision rates in the presentday Kuiper Belt and Centaurs regions: Application to surface activation and modification on Comets, Kuiper Belt Objects, Centaurs and Pluto-Charon. Icarus, 145, 220-229.

Ehrenfreund P., Glavin D. P., Botta O., Cooper G., and Bada J. L. (2001) Extraterrestrial amino acids in Orgueil and Ivuna: Tracing the parent body of CI type carbonaceous chondrites. Proc. Natl. Acad. Sci., 98, 2138-2141.

Emery J. P. and Brown R. H. (2001) Near-infrared spectroscopy of Trojan asteroids: Implications for the evolution of the solar system (abstract). In Lunar and Planetary Science XXXII, pp. 1385-1396. Lunar and Planetary Institute, Houston.

Erikson A. (2000) The present distribution of asteroid spin vectors and its relevance to the origin and evolution of main belt asteroids. Ph.D. thesis, Freie Universitaet, Berlin. (DLR Research Report 2000-37, ISSN \#1434-8454.)

Evans N. W. and Tabachnik S. A. (2000) Asteroids in the inner solar system II: Observable properties. Mon. Not. R. Astron. Soc., 319, 80-94.

Farinella P., Paolicchi P., and Zappalà V. (1981) Analysis of the spin rate distribution of asteroids. Astron. Astrophys., 104, 159165.

Fernandez Y. R., Jewitt D. C., and Shepard S. S. (2002) Thermal properties of Centaurs Asbolus and Chiron. Astron. J., 123, 1050-1055.

Fitzsimmons A., Dahlgren M., Lagerkvist C.-I., Magnusson P., and Williams I. P. (1994) A spectroscopic survey of D-type asteroids. Astron. Astrophys., 282, 634-642.

Foster M. J., Green S. F., and McBride N. (1999) Detection of water ice on 2060 Chiron. Icarus, 141, 408-410.

Fulchignoni M., Barucci M. A., Di Martino M., and Dotto E. (1995) On the evolution of the asteroid spin. Astron. Astrophys., 299, 929-932.

Gradie J. and Veverka J. (1980) The composition of the Trojan asteroids. Nature, 283, 840-842.

Gutierrez P. J., Ortiz J. L., Alexandrino E., Roos-Serote M., and Doressoundiram A. (2001) Short term variability of Centaur 1999 UG $_{5}$. Astron. Astrophys., 371, L1-L4.

Hahn G. and Bailey M. E. (1990) Rapid dynamical evolution of giant comet Chiron. Nature, 348, 132-136.

Hartmann W. K. (1980) Surface evolution of two-component stone/ice bodies in the Jupiter region. Icarus, 44, 441-453.

Hartmann W. K. and Cruikshank D. P. (1978) The nature of Trojan asteroid 624 Hektor. Icarus, 36, 353-366.

Hartmann W. K. and Cruikshank D. P. (1980) Hektor, the largest highly elongated asteroid. Science, 207, 976-977.

Hartmann W. K., Tholen D. J., Goguen J., Binzel R. P., and Cruikshank D. P. (1988) Trojan and Hilda asteroid lightcurves. I. Anomalously elongated shapes among Trojans (and Hildas?). 
Icarus, 73, 487-498.

Hartmann W. K., Tholen D. J., Meech K. J., and Cruikshank D. P. (1990) Chiron: Colorimetry and cometary behavior. Icarus, 83, $1-15$.

Hiroi T., Zolensky M. E., and Peters C. M. (2001) The Tagish Lake meteorite: A possible sample from a D-type asteroid. Science, 293, 2234-2236.

Hoffmann M., Fink U., Grundy W. M., and Hicks M. (1993) Photometric and spectroscopic observations of 5145 Pholus. J. Geophys. Res., 98, 7403-7407.

Jedicke R. J. and Herron J. D. (1997) Observational constraints on the Centaur population. Icarus, 127, 494-507.

Jewitt D. and Kalas P. (1998) Thermal observations of Centaur 1997 CU $_{26}$. Astrophys. J. Lett., 499, L103-L109.

Jewitt D. C. and Luu J. X. (1990) CCD spectra of asteroids. II. The Trojans as spectral analogs of cometary nuclei. Astron. J., 100, 933-944.

Jewitt D. C. and Luu J. X. (2001) Colors and spectra of Kuiper Belt objects. Astron. J., 122, 2099-2114.

Jewitt D., Luu J., and Chen J. (1996) The Mauna Kea-CerroTololo (MKCT) Kuiper Belt and Centaur survey. Astron. J., 112, 1225-1236.

Jewitt D. C., Trujillo C. A., and Luu J. X. (2000) Population and size distribution of small jovian Trojan asteroids. Astron. J., 120, 1140-1147.

Jones T. D., Lebofsky L. A., Lewis J. S., and Marley M. S. (1990) The composition and the origin of the $\mathrm{C}, \mathrm{P}$, and $\mathrm{D}$ asteroids: Water as a tracer of thermal evolution in the outer belt. Icarus, 88, 172-192.

Kern S. D., McCarthy D. W., Buie M. W., Brown R. H., Campins H., and Rieke M. (2000) Compositional variation on the surface of Centaur 8405 Asbolus. Astrophys. J. Lett., 542, L155L159.

Khare B. N., Bakes E. L. O., Cruikshank D. P., and McKay C. P. (2001) Solid organic matter on outer solar system bodies. Adv. Space Res., 27, 299-307.

Kissel J. and Krueger F. R. (1987) The organic component in dust from comet Halley as measured by the PUMA mass spectrometer on board Vega 1. Nature, 326, 755-760.

Kowal C. T. (1978) Surprise in the solar system. The Sciences, $18,12-15$.

Irvine W. M., Schloerb F. P., Crovisier J., Fegley B. Jr., and Mumma M. J. (2000) Comets: A link between interstellar and nebular chemistry. In Planets and Protostars IV (V. Mannings et al., eds.), pp. 1159-1200. Univ. of Arizona, Tucson.

Larsen J. A., Gleason A. E., Danzl N. M., Descour A. S., McMillan R. S., Gehrels T., Jedicke R., Montani J. L., and Scotti J. V. (2001) The spacewatch wide-area survey for bright Centaurs and Trans-Neptunian objects. Astron. J., 121, 562-579.

Lazzarin M., Barbieri C., and Barucci M. A. (1995) Visible spectroscopy of dark, primitive asteroids. Astron. J., 110, 30583072.

Lazzaro D., Florckzac M. A., Betzler A., Winter O. C., GiuliattiWinter S. M., Angeli C. A., and Foryta D. W. (1996) 2060 Chiron back to a minimum of brightness. Planet. Space Sci., 44, 1547-1550.

Lazzaro D., Florckzac M. A., Angeli C. A., Carvano J. M., Betzler A. S., Casati A. A., Barucci M. A., Doressoundiram A., and Lazzarin M. (1997) Photometric monitoring of 2060 Chiron's brightness at perihelion. Planet. Space Sci., 45, 1607-1614

Levison H. and Duncan M. (1997) From the Kuiper Belt to Jupiter-family comets: The spatial distribution of ecliptic comets. Icarus, 127, 13-32.
Levison H. F. and Weissman P. R. (1999) The Kuiper Belt. In Encyclopedia of the Solar System (P. R. Weissman et al., eds.), pp. 557-583. Academic, San Diego.

Levison H., Shoemaker E. M., and Shoemaker C. S. (1997) The dispersal of the Trojan asteroid swarm. Nature, 385, 42-44.

Levison H., Dones L., and Duncan M. (2001) The origin of Halley-type comets: probing in the inner Oort cloud. Astron. J., 121, 2253-2267.

Licandro J., Oliva E., and Di Martino M. (2001) Astron. Astrophys., 373, 29L-32L.

Lunine J. I., Owen T. C., and Brown R. H. (2000) The outer solar system: Chemical constraints at low temperatures on planet formation. In Planets and Protostars IV (V. Mannings et al., eds.), pp. 1055-1080. Univ. of Arizona, Tucson.

Luu J. X. (1993) Cometary activity in distant comets: Chiron. Publ. Astron. Soc. Pac., 105, 946-950.

Luu J. X. and Jewitt D. (1990) Cometary activity in 2060 Chiron. Astron. J., 100, 913-931.

Luu J. X. and Jewitt D. (1996) Color diversity among the Centaurs and Kuiper Belt objects. Astron. J., 112, 2310-2318.

Luu J. X. and Jewitt D. (1998) Optical and infrared reflectance spectrum of Kuiper Belt object $1996 \mathrm{TL}_{66}$. Astrophys. J. Lett., 494, L117-121.

Luu J., Jewitt D., and Cloutis E. (1994) Near-infrared spectroscopy of primitive solar system objects. Icarus, 109, 133-144.

Luu J. X., Jewitt D. C., and Trujillo C. (2000) Water ice in 2060 Chiron and its implications for Centaurs and Kuiper Belt objects. Astrophys. J. Lett., 531, L151-L154.

Marcialis R. L. and Buratti J. (1993) CCD photometry of 2060 Chiron in 1985 and 1991. Icarus, 104, 234-243.

Marsden B. (2001) List of Centaurs and Scattered-Disk Objects. http://cfa-www.harvard.edu/iau/lists/Centaurs.html.

Marzari F. and Scholl H. (1998) The growth of Jupiter and Saturn and the capture of Trojans. Astron. Astrophys., 339, 278-285.

Marzari F., Scholl H., and Farinella P. (1996) Collision rates and impact velocities in the Trojan asteroid swarms. Icarus, 119, 192-201.

Meech K. J. and Belton M. J. S. (1989) (2060) Chiron. IAU Circular 4770.

Milani A. (1994) The dynamics of the Trojan asteroids. In Asteroids Comets Meteors 1993, Proceedings IAU Symposium 160 (A. Milani et al., eds.), pp. 159-174. Kluwer, Dordrecht.

Moroz L. V., Arnold G., Korochantsev A. V., and Wäsch R. (1998) Natural solid bitumens as possible analogs for cometary and asteroid organics. Icarus, 134, 253-268.

Oikawa S. and Everhart R. (1979) The past and future orbit of 1977 UB, object Chiron. Astron. J., 84, 134-139.

Owen T. C., Cruikshank D. P., Dalle Ore C. M., Geballe T. R., Roush T. L., and de Bergh C. (1999) Detection of water ice on Saturn's satellite Phoebe. Icarus, 140, 379-382.

Parker J. M., Stern A., and Festou M. C. (1997) Ultraviolet observations of Chiron with the HST/FOS. Examining a Centaur's gray matter. Astron. J., 113, 1899-1908.

Peixinho N., Lacerda P., Ortiz J. L., Doressoundiram A., RoosSerote M., and Gutierrez P. J. (2001). Photometric study of Centaurs 10199 Chariklo (1997 $\left.\mathrm{CU}_{26}\right)$ and $199 \mathrm{UG}_{5}$. Astron. Astrophys., 371, 753-759.

Prialnik D., Brosch N., and Ianovici D. (1995) Modelling the activity of 2060 Chiron. Mon. Not. R. Astron. Soc., 276, 11481154.

Reitmeijer F. J. M. and Nuth J. A. (2000) Metastable eutectic equilibrium brought down to Earth. Eos Trans. AGU, 81(36), 409, 414-415. 
Reitmeijer F. J. M. and Nuth J. A. (2001) Serpentine by hydrogenation of Fe-rich ferromagnesiosilica PCs in aggregate IDPs (abstract). In Lunar and Planetary Science XXXII, Abstract \#1219. Lunar and Planetary Institute, Houston (CD-ROM).

Romon-Martin J., Barucci M. A., de Bergh C., and Peixinho N. (2001) Spectroscopy of Centaurs Asbolus and Chiron: Observations over full rotational period. Bull. Am. Astron. Soc., 33, 1034.

Sheppard S. S., Jewitt D. C., Trujillo C. A., Brown M. J. I., and Ashley M. C. B. (2000) A wide-field CCD survey for Centaurs and Kuiper Belt Objects. Astron. J., 120, 2687-2694.

School H. (1979) History and evolution of Chiron's orbit. Icarus, 40, 345-349.

Thompson W. R., Murray B. G. J. P. T., Khare B. N., and Sagan C. (1987) Coloration and darkening of methane clathrate and other ices by charged particle irradiation: Applications to the outer solar system. J. Geophys. Res., 92, 14933-14947.

Tholen D. J. and Barucci M. A. (1989) Asteroid taxonomy. In Asteroids II (R. P. Binzel et al., eds.), pp. 298-315. Univ. of Arizona, Tucson.
Tholen D. J., Hartmann W. K., and Cruikshank D. P. (1988) 1980 $P A$ and 1985 DO2. IAU Circular 4770.

Vilas F., Larson S. M., Hatch E. C., and Jarvis K. S. (1993) CCD reflectance spectra of selected asteroids. II. Low-albedo asteroid spectra and data extraction techniques. Icarus, 105, 67-78.

Weissman P. R. and Levison H. F. (1997) The population of the trans-neptunian region: The Pluto-Charon environment. In Pluto and Charon (S. A. Stern and D. J. Tholen, eds.), pp. 559-604. Univ. of Arizona, Tucson.

Womack M. and Stern S. A. (1995) 2060 Chiron $=$ Comet 95P/ Chiron. IAU Circular 6193.

Zappalà V., Di Martino M., Cellino A., Farinella P., De Sanctis G., and Ferreri W. (1989) Rotational properties of outer belt asteroids. Icarus, 82, 354-368.

Zellner B., Tholen D. J., and Tedesco E. F. (1985) The eight-color asteroid survey: Results for 589 minor planets. Icarus, 61, $355-416$. 